\title{
Análisis dialógico del discurso: posiciones ideológicas que movilizan la toma de decisiones curriculares de una profesora de educación básica
}

\author{
Dialogical Analysis of Discourse: Ideological positions that mobilize curriculum \\ decisions of a basic education teacher
}

\section{Análise dialógica do discurso: posicionamentos ideológicos que mobilizam a tomada de decisão curricular de um professor da educação básica}

\author{
Tamara Paola Caballero Guichard \\ Pontificia Universidad Católica de Chile, Chile \\ tpcaballero@uc.cl \\ https://orcid.org/0000-0002-6462-0909
}

\section{Resumen}

Las decisiones curriculares de los docentes sirven para dotar de pertenencia a lo que se enseña en las escuelas, pues le permiten al profesor adaptar o reconstruir el currículo prescrito externamente según la situación de cada aula. Sin embargo, las decisiones curriculares no son neutrales ni mecánicas; por el contrario, las posiciones ideológicas que poseen los profesores, así como otros factores políticos y socioculturales, contribuyen a que los docentes desarrollen determinadas aproximaciones de toma de decisiones curriculares en vez de otras. Considerando esto, el objetivo de este estudio fue evidenciar las posiciones ideológicas que movilizan la toma de decisiones curriculares a nivel de aula de una profesora de educación básica. Para ello, se aplicó el modelo de análisis dialógico del discurso a una entrevista semiestructurada realizada a la referida docente. Los resultados de esta investigación permiten identificar que la toma de decisiones curriculares de la profesora se mueve alrededor de un núcleo ideológico común, que es la flexibilidad curricular, la cual se muestra condicionada por tres aspectos: la propia 




Revista lberoamericana para la Investigación y el Desarrollo Educativo

ISSN $2007-7467$

experiencia docente, el diseño del currículo nacional y las condiciones de la institución educativa. De tal forma que al ser una profesora novel, que trabaja con un currículo que califica como sobrecargado y altamente prescriptivo, en el contexto de una institución enfocada en el cumplimiento y los resultados, predomina en su discurso una posición ideológica más rígida y técnica respecto de su toma de decisiones curriculares, que tienden a ser más reproductivas que adaptativas y constructivas.

Palabras clave: análisis del discurso, currículo escolar, decisiones curriculares, práctica docente.

\section{Abstract}

The teachers' curriculum decisions allow them to give pertinence to what is taught in the schools, because through these decisions the teachers can adapt or reconstruct the externally prescribed curriculum according to the situation of each classroom. However, curriculum decisions are neither neutral nor mechanical, on the contrary the ideological positions held by teachers, like other political and sociocultural factors, contribute to develop certain teacher approaches to curriculum decision-making. Considering this, the purpose of this study was to demonstrate the ideological positions in teachers discourse that mobilize curriculum decision-making at the classroom level in the case of a basic education teacher. To this end, the Dialogical Analysis of the Discourse model was applied to a semi-structured interview conducted with this teacher. The results of this research make it possible to identify that the teacher's curriculum decision-making moves around a common ideological core that is curricular flexibility, which is conditioned by three aspects: the teacher's own experience, the design of the national curriculum and the conditions of the educational institution. As conclusion, in the discourse of the studied teacher, as a novice teacher, who works with a curriculum that qualifies as overloaded and highly prescriptive, in the context of an institution focused on compliance and results, prevails a more rigid an technical ideological position when making curriculum decisions, which tend to be more reproductive than adaptive and constructive.

Keywords: discourse analysis, school curriculum, curriculum decisions, teaching practice. 




Revista Iberoamericana para la Investigación y el Desarrollo Educativo

ISSN 2007 - 7467

\section{Resumo}

As decisões curriculares dos professores servem para dar apropriação ao que é ensinado nas escolas, visto que permitem ao professor adaptar ou reconstruir o currículo prescrito externamente de acordo com a situação de cada sala de aula. No entanto, as decisões curriculares não são neutras nem mecânicas; pelo contrário, as posições ideológicas dos professores, bem como outros fatores políticos e socioculturais, contribuem para que os professores desenvolvam certas abordagens curriculares de decisão ao invés de outras. Diante disso, o objetivo deste estudo foi demonstrar os posicionamentos ideológicos que mobilizam a tomada de decisão curricular em nível de sala de aula de um professor da educação básica. Para tanto, aplicou-se o modelo de análise dialógica do discurso a uma entrevista semiestruturada com a referida professora. Os resultados desta pesquisa permitem identificar que a tomada de decisão curricular do professor gira em torno de um núcleo ideológico comum, que é a flexibilidade curricular, que é condicionada por três aspectos: a própria experiência docente, o desenho do currículo nacional. e as condições da instituição de ensino. Para que por ser uma professora iniciante, que trabalha com um currículo que se qualifica como sobrecarregado e altamente prescritivo, no contexto de uma instituição voltada para a conformidade e resultados, prevalece em seu discurso um posicionamento ideológico mais rígido e técnico suas decisões curriculares, que tendem a ser mais reprodutivas do que adaptativas e construtivas.

Palavras-chave: análise do discurso, currículo escolar, decisões curriculares, prática docente. Fecha Recepción: Mayo 2020 Fecha Aceptación: Octubre 2020

\section{Introducción}

Un asunto central de la política curricular de varios países —entre ellos Chile- es la flexibilidad y la autonomía curricular, entendidos como conceptos que permiten hacer más pertinentes los proyectos educativos nacionales (Bellei y Morawitz, 2016). En este contexto es donde las decisiones cotidianas del docente sobre el currículo se vuelven un tema relevante, ya que es precisamente a través de sus decisiones curriculares que se contextualiza constantemente la propuesta nacional haciéndola más pertinente (Espinoza, Riquelme y Salas, 2017).

Sin embargo, la toma de decisiones curriculares que realiza el docente no es un proceso neutral, mecánico o instrumental (Pinar, 2014). En cambio, representa un proceso subjetivo e intersubjetivo, político y técnico-pedagógico en el que se presentan y se entrecruzan posiciones 


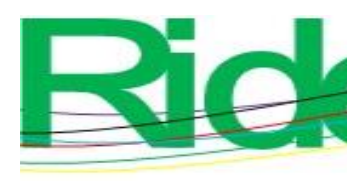

Revista Iberoamericana para la Investigación y el Desarrollo Educativo

ISSN $2007-7467$

ideológicas (Cox, 2018; Magendzo, 2018). De acuerdo con Schiro (2013), las ideologías sobre el currículo escolar contribuyen a una determinada aproximación de toma de decisiones curriculares por el docente. Esto se hace más evidente a nivel micro (es decir, el aula), donde el docente es el actor clave, a partir de lo que planifica para su clase y de la interacción con los alumnos (Pang, 2012). Por eso, es posible considerar que según la ideología a la que él se adhiere y según ciertas condiciones de decisión y actuación, decide reproducir, adaptar o reconstruir el discurso oficial y prescrito como parte de una danza en la que se fluye entre diferentes opciones y mundos curriculares (Aoki, 2005). De acuerdo con distintos autores, dentro de esta danza de opciones curriculares, existen unas que favorecen la mejora de los aprendizajes de los alumnos y se relacionan con la adaptación y construcción curricular desde la interacción áulica (Remillard y Heck, 2014; Schultz, 2011; Shawer, 2010).

Sin embargo, como indican Greany y Waterhouse (2016), no basta con que la política pública declare ciertas posturas sobre lo curricular; se trata de todo un conjunto de aspectos y condiciones determinantes para asegurar que autonomía y desarrollo curricular realmente se materialicen en las decisiones del docente en su día a día.

De hecho, en el contexto chileno, Caro y Aguilar (2018) señalan que, a pesar de su relevancia en la discusión política y su complejidad, las decisiones curriculares del docente en relación con la autonomía, flexibilidad y pertinencia del currículo han sido abordadas en la mayoría de los casos desde la tensión entre una alta o baja prescripción en el diseño de la política curricular. Además, han sido asumidas en la naturaleza de un quehacer docente que ha permanecido relegado casi exclusivamente a la instrucción en el sentido más operativo y técnico $\mathrm{y}$, desde el punto de vista curricular, a la implementación eficaz, desde lo prescriptivo y burocrático (Pinar, 2014).

Aunado a esto, en la revisión de la literatura, Handler (2010) muestra — sobre la toma de decisiones curriculares docentes a nivel internacional, entre los años 2000 y 2010 - que estos estudios se han centrado mayormente en la parte preactiva del ejercicio decisional de los profesores (planificaciones) y lo han abordado desde un análisis documental, dejando en segundo plano la práctica discursiva del docente que permite comprender esta toma de decisiones curriculares, e incluso dejando de lado la misma práctica curricular interactiva en el interior del salón de clases. Un panorama similar se observa en la producción nacional chilena sobre este tema, cuya mayor producción se encuentra entre los años 2000 y 2007, y se centra 


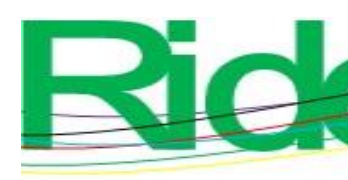

Revista Iberoamericana para la Investigación y el Desarrollo Educativo

ISSN $2007-7467$

mayoritariamente en datos recogidos desde una perspectiva documental. Los resultados de estas investigaciones chilenas indican que la mayoría de las veces los profesores se quedan en un nivel de decisión reproductivo y que cuando estos profesores toman decisiones de adaptación, estas se refieren solo a agregar elementos al currículo prescrito (Guzmán, Meza, Pascual y Pinto, 2007; Meza, Pascual y Pinto, 2006). Es decir, se ha encontrado en los estudios precedentes que predomina un nivel de autonomía curricular bajo o nulo en los docentes.

Mencionado lo anterior, cabe plantearse las siguientes interrogantes: ¿qué posiciones ideológicas movilizan las decisiones curriculares de los docentes? ¿Cuáles son las tensiones que se manifiestan en los discursos de los docentes sobre su toma de decisiones curriculares? A partir de estas preguntas, se estableció como objetivo evidenciar las posiciones ideológicas que movilizan la toma de decisiones curriculares a nivel de aula de una profesora de educación básica de una escuela municipal de la Región Metropolitana de Santiago Chile.

Considerando este objetivo, se pretendió mirar las decisiones curriculares del docente como procesos que no suceden a priori, sino bajo ciertas condiciones y desde la complejidad de una práctica cargada de sentidos y de ideologías que se gestionan en la inmediatez del día a día. Lo anterior contribuye a la comprensión de factores asociados con ciertas aproximaciones curriculares que favorecen en mayor medida el aprendizaje de los alumnos. Esto, además, aporta elementos para reconceptualizar el rol docente frente a las nuevas demandas curriculares expresas en la política educativa actual, desde las cuales se discutan las condiciones y orientaciones para ello desde los conceptos propuestos de autonomía y flexibilidad curricular.

\section{La reconceptualización del currículo: punto de partida para mirar las decisiones curriculares}

Existe una mirada instrumentalista predominante sobre el currículo y los diferentes procesos y categorías implicadas, lo cual lo reducen a un producto o a un proceso burocrático (Pinar, 2014). Ante esto, la corriente reconceptualista del currículo responde desde una mirada fenomenológica, afirmando que el currículo no es un producto ni un simple proceso, sino que se trata de una experiencia vivida, una práctica discursiva compleja (Aoki, 2005; Pinar, 2014). En tal sentido, cuando Pinar (2014) reconoce el currículo como una conversación compleja, no se refiere a esta en términos genéricos de lo que es una conversación, sino que lo pone en el ámbito de lo dialogal, como práctica discursiva. 


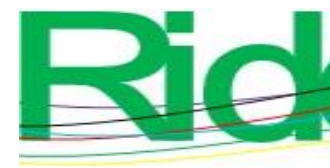

Revista Iberoamericana para la Investigación y el Desarrollo Educativo

ISSN $2007-7467$

En otras palabras, se trata de una práctica cargada de significados en un mundo particular y en una temporalidad que supone una co-pertenencia de pasado, presente y futuro (Pinar, 2014). Desde la mirada discursiva del currículo, Díaz Barriga (2010) da cuenta de este como un espacio dialógico donde actores educativos negocian el poder. Esto significa que al ser donde se definen y debaten fines, contenidos de lo que se enseña, se trata de un campo en pugna y en tensión.

En este trabajo se conceptualiza esta mirada precisamente desde esta reconceptualización, pues desde aquí se reconocen en el currículo las dimensiones de alteridad e intersubjetividad que se implican en él, desde categorías biográficas, políticas, históricas y estéticas que ponen al sujeto y al contexto en el centro (Pinar, 2014).

Dentro del ámbito escolar, el docente en esta corriente, como actor curricular, es visto como sujeto entrecruzado por diferentes diálogos, por sus historias de vida, su trayectoria, su experiencia personal y profesional en un lugar y tiempo determinado.

\section{Las decisiones curriculares del docente a nivel aula}

Desde la mirada curricular asumida en el apartado anterior es que resulta apropiado hablar sobre las decisiones curriculares del docente reconociendo la complejidad de su quehacer curricular cotidiano, que tiene lugar entre la tensión de la rutina y la innovación (Pinar, 2014). Las decisiones sobre el currículo representan seleccionar cuál es el conocimiento curricular, es decir, cuáles son los cocimientos, aprendizajes y las competencias que se deben lograr (Magendzo, 2018).

Considerando esto, el nivel micro de concreción curricular (el aula) se vuelve especialmente importante, pues es donde el docente está en interacción directa con los alumnos (Pang, 2012 ). En consecuencia, ciertas decisiones curriculares en su planificación y dentro del aula los llevan a desarrollar diferentes experiencias de aprendizaje que impactan a su vez de manera diferente en los alumnos (Craig, 2012; Doyle y Rosemartin, 2012; Schultz, 2011).

De acuerdo con Aoki (2005), en el aula las decisiones curriculares del docente se sitúan en una especie de tierra media: entre las pretensiones prescritas de otros niveles curriculares (políticos, institucionales) y lo que pasa en la interacción en el aula. Por eso, las aproximaciones curriculares que los docentes tienen frente a los instrumentos curriculares prescritos, en términos de la autonomía de sus decisiones, pueden considerarse reproductivas, adaptativas o constructivas (Guzmán, 2011; Shawer, 2010). Reproductivas si la mayoría de las veces se apegan 


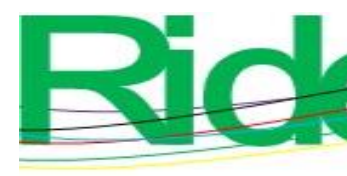

Revista Iberoamericana para la Investigación y el Desarrollo Educativo

ISSN $2007-7467$

o tratan de apagarse exclusivamente a los instrumentos curriculares prescritos; adaptativas si incluyen otros elementos y modifican lo prescrito en relación con las interacciones con los alumnos y su propio criterio, pero sin perder el eje orientador general; y constructivas si crean nuevos elementos con independencia de los instrumentos curriculares prescritos y en relación directa con las interacciones del aula. De acuerdo con distintos autores (Craig, 2012; Doyle y Rosemartin, 2012; Shawer, 2010), las decisiones de los docentes que permiten el desarrollo curricular y que generan experiencias educativas más pertinentes y significativas son aquellas que se mueven entre las aproximaciones adaptativas y constructivas, en tanto representan que el profesor interpreta y responde a las peculiaridades y demandas de los propios textos y sujetos.

Cox (2018) y Magendzo (2018) consideran que tomar una decisión de un tipo u otro constituye un proceso subjetivo, político y técnico-pedagógico porque se da en el interior de un contexto personal, social, económico, cultural y educacional, en el que se involucran intereses, tendencias y posiciones ideológicas y conocimientos disciplinares y pedagógicos diversos. Para Boschman, McKenney y Voogt (2014) la toma de decisiones curriculares de los profesores está influenciada por las orientaciones existentes (ideologías, conocimiento técnico, creencias y prácticas), prioridades externas (exigencias institucionalidades, normativas), y preocupaciones prácticas (lo que es posible y alcanzable dentro de los límites de la práctica).

\section{Las ideologías como movilizadoras de las decisiones curriculares}

Para Schiro (2013) los actores que participan de la negociación curricular en el espacio escolar lo hacen desde distintas creencias, intereses y visiones del mundo, de la educación y específicamente del currículo. Estos pueden ser concebidos como ideologías, que se refieren a esquemas de pensamiento sobre el conocimiento, la enseñanza y el aprendizaje, que permean la visión de los sujetos sobre el currículo y se relacionan con particulares formas de acción, prácticas y actitudes de los profesores (Schiro, 2013).

Para Voloshinov (2009) la ideología "es parte de una realidad natural o social no solo como un desarrollo físico, un instrumento de producción o un producto de consumo, sino que, además, a diferencia de los fenómenos enunciados, refleja y refracta una realidad distinta que está más allá de su materialidad. Todo producto ideológico posee una significación” (p. 31). Para Bajtín (1981) la ideología se refiere a "un sistema de ideas, en cuanto sistema semiótico, en el 


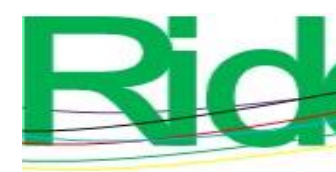

Revista Iberoamericana para la Investigación y el Desarrollo Educativo

ISSN $2007-7467$

sentido de que involucra el intercambio concreto de signos en la sociedad y en la historia, en el que cada palabra/discurso delata la ideología de su hablante” (p. 429).

Podríamos decir entonces, en el ámbito de lo curricular, que las ideologías o posicionamientos sobre el currículo a los que los profesores se adhieren interpelan y condicionan su forma de relacionarse con el currículo; es decir, pensamiento, lenguaje y acción no se encuentran disociados.

\section{Método}

Considerando que las palabras delatan la ideología de su hablante (Bajtín, 1981), se hace necesario analizar el discurso de los profesores sobre sus decisiones curriculares desde una perspectiva dialógica. Para ello, en este trabajo se ha asumido una postura sociocultural sobre el discurso, en la que se considera que este solo puede comprenderse en su complejidad cuando se entiende como un fenómeno sociológico, histórico e ideológico llevado a cabo a través de la enunciación que surge de la comunicación verbal concreta (Volóchinov, 2009), poniendo además en el centro de las aproximaciones al dialogismo, el cual permite repensar las relaciones de significado desde la contextualización y la relación con los otros (Ávila y Medina, 2012).

Por ende, y desde la postura sociocultural, el análisis dialógico del discurso (ADD) fue considerado como el más adecuado para analizar las ideologías impresas en el discurso de los docentes sobre las decisiones curriculares. Este permite visibilizar las distintas voces o posicionamientos incorporados en el discurso y analizar el posicionamiento socioideológico que lo sustenta, así como los planos subjetivos presentes en él (Ávila y Medina, 2012).

El ADD aquí planteado se basa en la propuesta de enfoque de la teoría de la enunciación (Ávila y Medina, 2012; Larraín y Medina, 2007), según la cual el enunciado representa la unidad de la comunicación discursiva, y se reconceptualizan las categorías de análisis enunciativo de la siguiente manera (Larraín y Medina, 2007):

- El sujeto de la enunciación es aquel aspecto que remite al acto de enunciación en sí mismo, es decir, el modo en que se expresa o inscribe el rol asumido en la enunciación, la cual puede ser contextualizada.

- El sujeto enunciado se refiere al centro de referencia ideológico desde el que se elabora lo enunciado. 




Revista Iberoamericana para la Investigación y el Desarrollo Educativo ISSN $2007-7467$

- El locutor se refiere al responsable material de la voz o voces en el discurso.

- El enunciador se refiere a las posiciones ideológicas presentes en el texto.

- El sujeto discursivo a la posición dominante que atraviesa el texto, la posición última, sostenida a través del recorrido textual /discursivo.

Considerando esta aproximación metodológica, se aplicó un ADD al corpus de una entrevista semiestructurada realizada a una profesora de educación básica de una escuela municipal de la comuna de Ñuñoa. La entrevista tuvo una duración de 61 minutos, fue grabada y posteriormente transcrita por el mismo entrevistador para su análisis.

De acuerdo con Bajtín (1981), el género discursivo se refiere a la forma que adquieren los enunciados en el uso concreto de la lengua dentro de una esfera de la práctica social y que establecen ciertas formas convencionales de participación. En este sentido, una de las características más evidentes del género de la entrevista de investigación tiene que ver con la diferencia de roles de la palabra de los interlocutores, pues a pesar de que puede haber un conocimiento y códigos compartidos sobre el objeto de la entrevista, el entrevistador juega un rol más instrumental y estratégico que busca - a través de la generación de preguntas dentro de un apartado de inicio, desarrollo y cierre - exhortar al entrevistado a entregar información, a desarrollar un razonamiento sobre algo o a hacerse cargo de un hecho discursivamente (Meneses y Rodríguez, 2011).

Particularmente se trató de una entrevista de investigación semiestructurada en el marco del proyecto de magíster titulado La toma de decisiones curriculares a nivel aula del docente de educación básica en el contexto municipal. Este proyecto consistió en un estudio de caso con el objetivo de caracterizar las decisiones curriculares del docente e identificar factores asociados a estas características. Para ello, se han recolectado datos de fuentes variadas: entrevistas, observación áulica, análisis de la planificación y bitácoras de clase. La entrevista que en el presente trabajo se analiza fue realizada a una docente novel de 26 años de edad, con estudios en Pedagogía Básica, mención en Ciencias Sociales, que actualmente es profesora-jefa de $2 .^{\circ}$ básico en una escuela municipal de la comuna de Ñuñoa, en la Región Metropolitana de Santiago Chile.

En la entrevista realizada a esta profesora, el entrevistador procuró elidir sus opiniones, juicios o valores para no alterar la información recabada. Además, vale acotar que existió un guion de entrevista con preguntas referentes a diferentes dimensiones del objeto de investigación, es decir, las decisiones curriculares cotidianas del docente en relación con los 


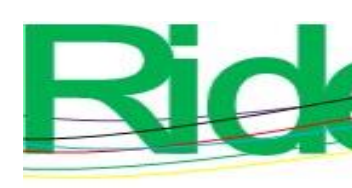

Revista Iberoamericana para la Investigación y el Desarrollo Educativo

ISSN $2007-7467$

instrumentos curriculares oficiales (bases curriculares, programas de las bases curriculares, libros de texto).

Específicamente - en el caso de este trabajo - el ADD se enfocó en las preguntas presentadas en el primer apartado: ¿qué posiciones ideológicas movilizan las decisiones curriculares de los docentes? ¿Cuáles son las tensiones que se manifiestan en los discursos de los docentes sobre su toma de decisiones curriculares? Las categorías de análisis en el ADD fueron el sujeto enunciado, los enunciadores y el sujeto discursivo (Larraín y Medina, 2007), ya que estos permiten identificar el centro ideológico del discurso, los diferentes posicionamientos o voces que tensionan la toma de decisiones y el posicionamiento que predomina.

Considerando estas categorías, las marcas o huellas discursivas pesquisadas fueron las siguientes: los deíticos de persona, tiempo y lugar, los subjetivemas y las modalidades de la enunciación. Los deíticos son considerados como la marca básica sobre la cual se identifican las categorías de la enunciación para el análisis, puesto que denotan la posición linguiística en que se posiciona el enunciador y las circunstancias espacio-temporales en que se produce el enunciado (Ávila y Medina, 2012). Los subjetivemas — siguiendo la propuesta de KerbratOrecchioni (2010) — son aquellas unidades en que el enunciador se revela explícitamente a través de valoraciones subjetivas en ítems de carácter nominal adjetivo, adverbial, verbal que tienen marcas afectivas, evaluativas o axiológicas. Finalmente, las modalidades de la enunciación se refieren a la manera en que el enunciador expresa su actitud hacia el contenido de lo que se comunica. Siguiendo la propuesta de Álvarez (2001, citado por Ávila y Medina, 2012), estas modalidades serían la alética (necesidad, posibilidad), deóntica (obligación, permiso, prohibición), epistémica (realidad mental), volitiva (voluntad) y apreciativa (valoración).

Por otro lado, dada la naturaleza del género discursivo analizado y las preguntas orientadoras, se llevó a cabo una segmentación que tuvo dos criterios: los momentos de la entrevista (inicio, desarrollo y cierre) y un criterio temático, que implicó segmentar aquellos fragmentos donde el entrevistado hiciera referencia al currículo nacional (bases curriculares) en su toma de decisiones cotidianas. A partir de estos criterios se obtuvieron nueve segmentos, seleccionados respetando los pares adyacentes propios de la entrevista (entrevistadorentrevistado): uno del inicio, seis del desarrollo o desarrollo y dos del cierre. De estos nueve segmentos, cuatro fueron seleccionados para conformar la muestra del análisis de este trabajo: 


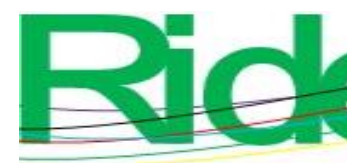

\section{Revista Iberoamericana para la Investigación y el Desarrollo Educativo \\ ISSN 2007 - 7467}

uno del inicio, uno del cierre y dos del desarrollo. Esta última selección consideró nuevamente tener la representación de todos los momentos de la entrevista y que estos segmentos fueran los más representativos de cada momento.

\section{Resultados}

A través del ADD realizado a los cuatro segmentos seleccionados — ver anexo 1—, se observa que la tensión ideológica se presenta alrededor de distintas variaciones de un centro ideológico común. Estas variaciones aparecen en función de las preguntas formuladas por el entrevistador y las categorías nominales a las que hacen referencia esas interrogantes. Por ello, es posible identificar que el sujeto del enunciado común es la flexibilidad curricular, es decir, la capacidad para tomar decisiones de adaptación y construcción sobre los objetivos y contenidos según lo prescrito en las bases curriculares. A partir de este sujeto del enunciado, se desarrolla el relato y se va tematizando en los diferentes fragmentos como se muestra en la figura 1:

Figura 1. Sujeto del enunciado (SE) y sus variaciones

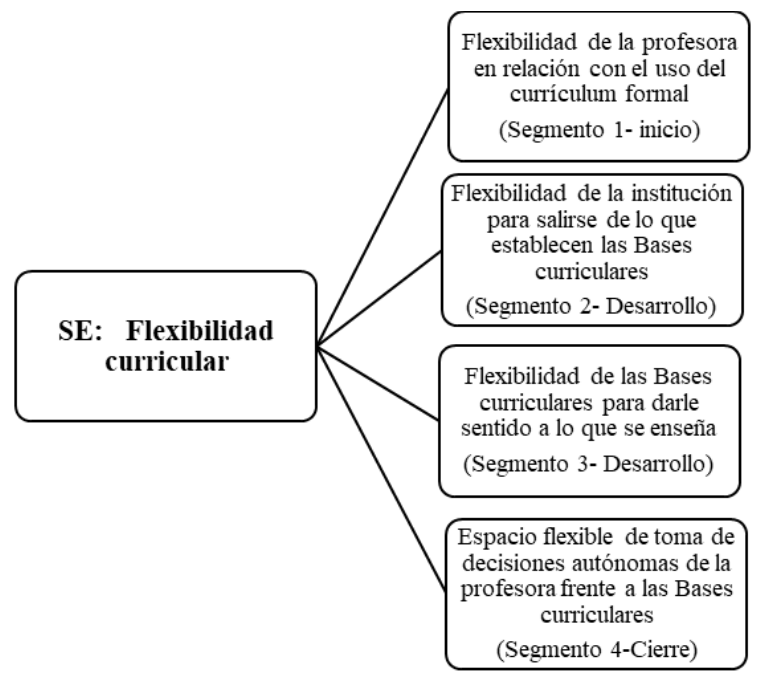

Fuente: Elaboración propia

Frente a este sujeto del enunciado se presenta una tensión ideológica en el discurso de la profesora entre dos enunciadores principales: El que expresa que existe flexibilidad para tomar decisiones de ajuste sobre el currículo nacional y un E2 que expresa que no existe flexibilidad para tomar decisiones de adaptación sobre el currículo nacional. Estos enunciadores (E1 y E2) se presentan en los fragmentos analizados con variaciones, que más que posicionamientos 


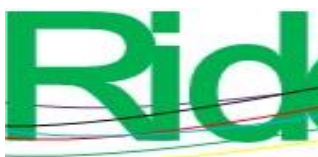

\section{Revista Iberoamericana para la Investigación y el Desarrollo Educativo \\ ISSN 2007 - 7467}

distintos que los conviertan en nuevos enunciadores, son argumentos distintos que sustentan a alguno de los dos posicionamientos. A continuación, se ofrecen dos figuras con los enunciadores y sus variaciones argumentativas:

Figura 2. Enunciador 1 (E1) y sus variaciones argumentativas

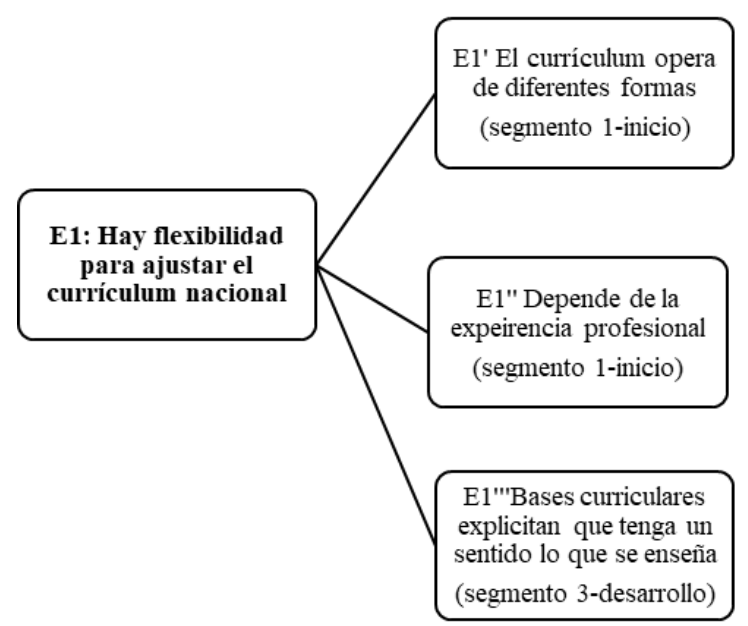

Fuente: Elaboración propia

Figura 3. Enunciador 1 (E2) y sus variaciones

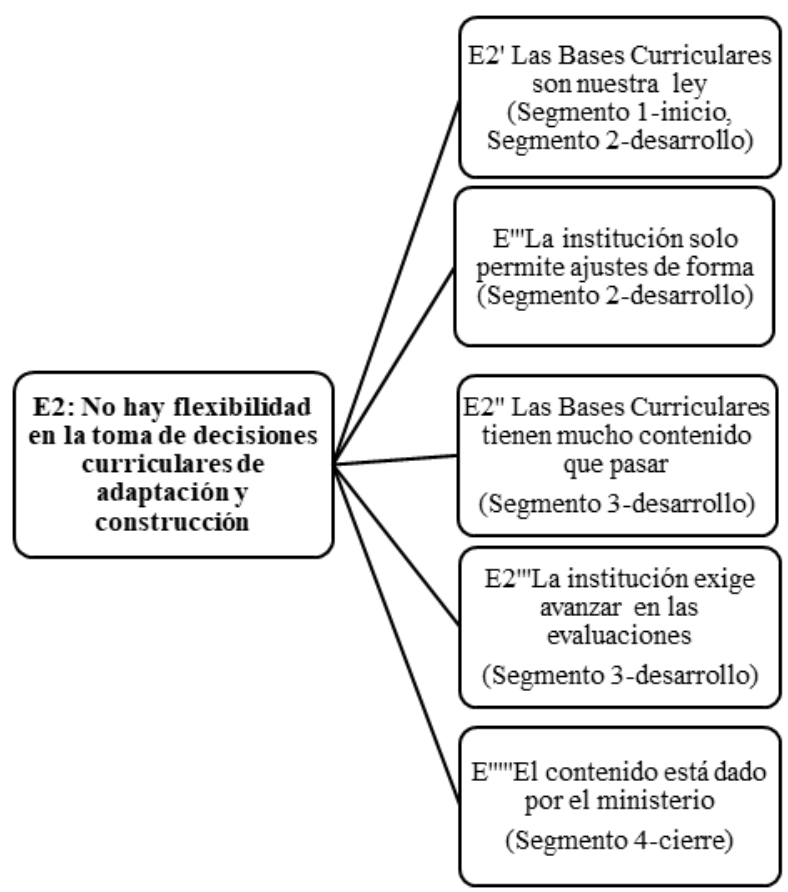

Fuente: Elaboración propia 


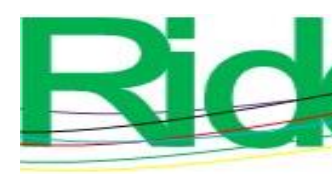

Revista Iberoamericana para la Investigación y el Desarrollo Educativo

ISSN 2007 - 7467

De acuerdo con el ADD realizado, el enunciador 1 fue identificado en los segmentos 1 y 3, y se caracteriza por tener una visión del currículo multidimensional, que no solo implica las bases curriculares a las que se refiere como currículo formal, sino que también reconoce el currículo informal y oculto que se crea en la relación con los alumnos; esto se inscribe de la siguiente manera: "la verdad que trabajar con el currículo no me complica// el currículum también opera de distintas formas (..)" (segmento 1- inicio, línea 16-19). Y en este sentido visualiza las posibilidades explícitas en el planteamiento general de las bases de sinergia entre el currículo formal y currículo informal para dar sentido al currículo que se enseña: "De cierta forma están basadas en que tenga un sentido y que tenga un aprendizaje significativo" (segmento 3-desarrollo, línea 6-9).

Además, este posicionamiento reconoce la necesidad de experiencia profesional para tomar decisiones que flexibilicen el currículo, que en el enunciado se ejemplifica como "pero a medida que uno ya se va desempeñando/ va conociendo a los chiquillos /y uno también se va conociendo" (segmento 1-inicio, línea 10-13). Por otro lado, es importante resaltar que en la tesis de este posicionamiento solo visualiza la flexibilidad curricular en términos de "hacer los ajustes necesarios" (segmento 1-inicio, línea 28), que representa un nivel bajo de adaptación curricular (Guzmán, 2011; Shawer, 2010).

Considerando esta caracterización del E1, se identificó que este se ubica dominantemente en dos modalidades: la epistemológica, inscrita en verbos cognitivos y sustantivos que demuestran ciertos niveles de certeza; y la alética, que evidencia una noción de necesidad a través de inscripciones como "que tenga" y "necesarios". En el caso de los subjetivemas en los que se inscribe, estos relativizan lo que se dice, implican progresión temporal o hacen referencia a la autonomía. En el caso de los deíticos personales, encontramos que la mayoría de las veces hay una inscripción impersonal e indeterminada a través del pronombre indeterminado uno. Solo una vez hay una inscripción desde el pronombre personal "yo", y esta se modaliza epistemológicamente. Además de esto, se identificaron pronombres reflexivos en el discurso en la primera persona. A continuación, se enseña la figura 4 con las huellas discursivas más representativas del enunciador, lo cual evidencia lo mencionado anteriormente: 


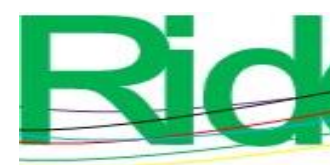

Revista Iberoamericana para la Investigación y el Desarrollo Educativo

ISSN $2007-7467$

Figura 4. Inscripción del E1

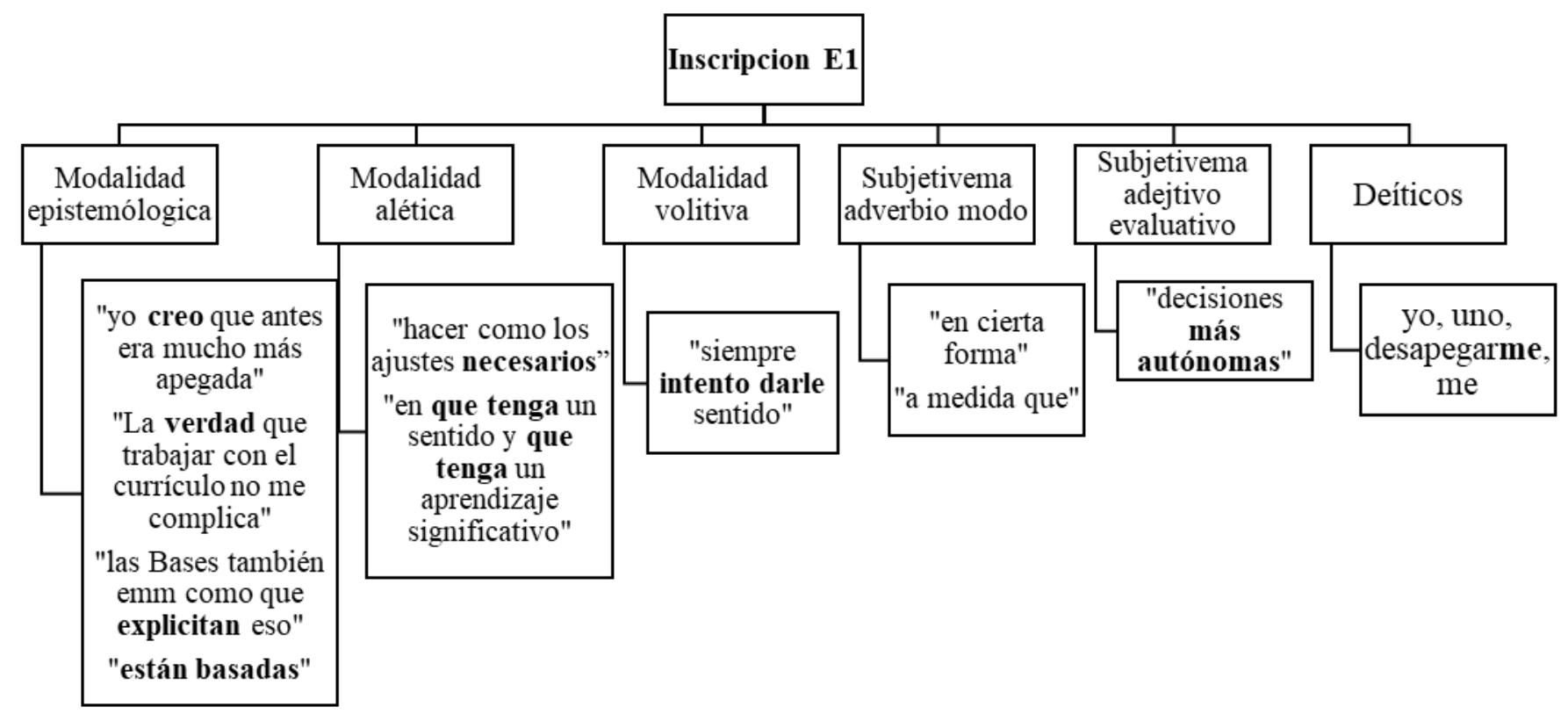

Fuente: Elaboración propia

Por otro lado, el enunciador 2 -identificado en los segmentos 1, 2, 3 y 4 - se caracteriza por tener una visión del currículo como producto a ser implementado, siendo este producto las bases curriculares. Esta visión del currículo solo da posibilidad a decisiones reproductivas, lo que se puede ejemplificar con la cita "entonces puede ser como un copy paste" (segmento 4cierre, línea 12), y está condicionada por la política curricular, como se observa en estas citas "porque es como nuestra ley y la que rige como los contenidos de las habilidades que uno va a trabajar" (segmento 1-inicio, línea 7-9), "por un tema como legal todo tiene que tributar al objetivo de aprendizaje" (segmento 2-desarrollo, línea 7-8).

El diseño y extensión del contenido de las bases curriculares también se muestran como una condición de no flexibilidad: "Pero también el contenido a veces que uno tiene que pasar/ uno no siempre logra darle esa / como ponerle ese sello a la unidad porque también uno tiene que cumplir con lo que se te exige // como que uno tiene que cumplir sí o sí con las bases y a veces es mucho contenido//" (segmento 3-desarrollo, línea 16-19). Finalmente, este enunciador de inflexibilidad se sostiene desde la exigencia de la escuela, como por ejemplo en la siguiente cita: "Y la verdad como que uno tiene que ir avanzando con las evaluaciones con lo que te pide el colegio entonces a veces uno se ve como coartado" (segmento 3-desarrollo, línea 23-25). 


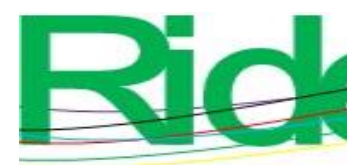

Revista Iberoamericana para la Investigación y el Desarrollo Educativo

ISSN $2007-7467$

A través del análisis se identificó que este enunciador se inscribe principalmente a través de la modalidad deóntica, haciendo referencia a la exigencia de reproducir fielmente el contenido de las bases curriculares, a la obligación de pasar el contenido. Además, se observa que existe una inscripción desde el pronombre indeterminado "uno", que relativiza y generaliza los argumentos sobre la inflexibilidad curricular y al mismo tiempo que dan cuenta de una voz perdida del sujeto que elabora el enunciado. Además, este pronombre indeterminado se acompaña de verbos pronominalizados en primera y tercera persona del singular (la mayoría además reflexivos), y en la primera persona del plural haciendo referencia al desarrollo docente, donde de nuevo el sujeto que elabora el enunciado se invisibiliza.

Encontramos también subjetivemas evaluativos que hacen referencia a la pesadez de tomar decisiones curriculares no reproductivas (mucho, muy difícil) y subjetivemas afectivos con adjetivos como "coartado" que denotan el sentir de la profesora. En este sentido, se reconoce la relación de exigencia entre la institución y el quehacer del docente limita su participación curricular a la reproducción de objetivos y contenidos y a hacer ajustes de forma, pero no que tengan que ver con modificar o añadir contenidos y menos con construirlos de forma propia, lo que se puede observar en el subjetivema adverbial utilizado en la siguiente cita: "Solo como de forma". Esto se vincula con la identidad del profesor que se configura en los límites del diseño de las actividades de aula, lo que se inscribe claramente con deícticos posesivos como "mi espacio" "de uno", que reafirman cuál es el lugar de autonomía y flexibilidad que reconoce como propio. A continuación, en la figura 5 se muestran las huellas discursivas más representativas del enunciador: 


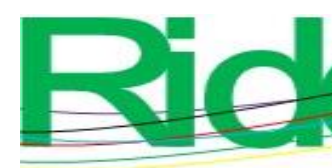

Revista Iberoamericana para la

Figura 5. Inscripción del E1



Fuente: Elaboración propia

A pesar de que existen estos dos enunciadores, por la manera en que se inscriben, es posible decir que la tensión ideológica que representan se resuelve a lo largo del discurso, en tanto E2 termina siendo más consistente y domina al E1 (figura 6). E1 se inscribe en un nivel de pensamiento, de necesidad y deseo del docente de esta flexibilidad según las modalidades pesquisadas, principalmente al inicio de la entrevista y al inicio de las respuestas, que emerge como una apropiación del discurso deseable sobre las bases curriculares, y donde recae el peso de la flexibilidad en el propio docente. Sin embargo, mientras va avanzando el discurso y se tematiza el núcleo ideológico en aspectos de su quehacer cotidiano es desplazado por el E2, conformado por las condiciones de obligatoriedad y exigencia de categorías nominales como las bases curriculares y la escuela; en consecuencia, E2 termina teniendo un peso mayor en el discurso, tanto por la presencia a lo largo de los segmentos de la modalidad deóntica que lo caracteriza como por el tipo de subjetivemas afectivos con los que se inscribe este enunciador, ligados a la manera en que la profesora siente y vive la toma de decisiones sobre el currículo, en una relación donde este enunciador es el que termina movilizando las decisiones curriculares del docente a un nivel de reproducción de lo prescrito en las bases curriculares. 

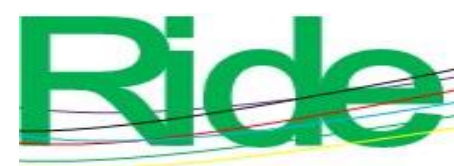

Revista Iberoamericana para la Investigación y el Desarrollo Educativo

Figura 6. Tensión dialógica

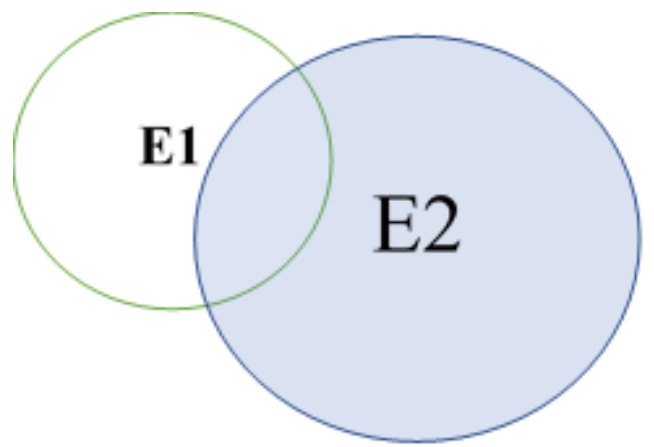

Fuente: Elaboración propia

Como se observa en la figura 6, el E2, el posicionamiento de que no hay flexibilidad curricular es el sujeto discursivo pesquisado en estos segmentos. Es decir, es el posicionamiento dominante que atraviesa los fragmentos analizados, desde los diferentes argumentos que lo sustentan, que están especialmente relacionados a factores externos al docente.

\section{Discusión}

Las categorías que tematizan el centro ideológico del discurso de la profesora sobre su toma de decisiones curriculares (es decir, la flexibilidad curricular) evidencian la complejidad de las decisiones curriculares, y las interrelaciones que se entretejen en ellas, tal como expresan Cox (2018), Magendzo (2018) y Boschman et al. (2014). Esto significa que las categorías identificadas están relacionadas con niveles distintos de desarrollo curricular (Pang, 2012) que se tensionan: la política curricular (nivel macro), gestión curricular de la institución (nivel meso), y la propia experiencia de la docente (nivel micro).

En medio de estas categorías alrededor de la flexibilidad curricular, la ideología de la profesora se tensiona entre una visión multidimensional y más flexible respecto del currículo nacional y una más rígida y técnica. En esta tensión termina predominando la segunda visión, especialmente influenciada por las condiciones contextuales que constriñen la toma de decisiones curriculares de la profesora. Estas condiciones contextuales limitan al profesor a lo que Aoki (2005) considera un papel de mero implementador. En tanto, esta posición ideológica más técnica del currículo — como señala Schiro (2013) — a su vez permea una aproximación más reproductiva que adaptativa y constructiva en sus prácticas curriculares (Shawer, 2017). En términos de Aoki (2005), termina dominando el mundo de la prescripción sobre el mundo del aula. 




Revista Iberoamericana para la Investigación y el Desarrollo Educativo

ISSN $2007-7467$

Finalmente, es importante resaltar que el análisis dialógico del discurso como modelo de estudio brinda la posibilidad de pesquisar las posiciones y voces en los discursos de los actores curriculares, con lo cual se recupera la complejidad de su actuación desde aspectos internos y externos que se tensionan y se materializan lingüísticamente en el discurso. En otras palabras, análisis como el presentado en este trabajo permiten trascender la identificación del tipo de prácticas curriculares dominantes — como se aborda en los estudios antecedentes (Guzmán et al., 2007; Meza et al., 2006) — para adentrarse en la comprensión de las posiciones que las movilizan.

\section{Conclusiones}

A partir del ADD realizado, se concluye que el núcleo ideológico del discurso sobre las decisiones curriculares se enfoca en la flexibilidad curricular, y que dicho eje se construye en el discurso analizado a través de tres grandes categorías, las cuales a su vez se componen de las siguientes subcategorías: la experiencia docente, el currículo nacional y las condiciones de la institución educativa.

Por otro lado, el ADD evidencia materialmente la presencia de dos posiciones que se contraponen en el discurso sobre las decisiones curriculares: una instaurada en lo necesario, en lo deseable que nace de los planteamientos y discursos generales de la política curricular de tipo más práctico, y otra en la práctica cotidiana que se ciñe en la exigencia, en la obligatoriedad y el cumplimiento de tipo más técnico, que no deja lugar a una toma de decisiones curriculares autónomas y centradas en la interacción de los sujetos y contextos. Esta última posición termina predominando, lo que evidencia una pérdida de voz de la profesora, que caracteriza todo del discurso. Se puede decir, por tanto, que se trata de una pérdida de sí misma como autora o agente del currículo, que reduce su identidad a las actividades de aprendizaje. Esto, además, demuestra la falta de condiciones reales para la flexibilidad y autonomía curricular del docente.

Finalmente, es necesario mencionar que el ADD realizado hizo posible acercarnos a los posicionamientos ideológicos en el discurso de la profesora, desde marcas lingüísticas concretas, que hace más riguroso el análisis del discurso y los resultados arrojados. De esta forma se superan enfoques que se limitan solo al contenido, como los usados en la mayor parte de estudios sobre este tema, los cuales tampoco aprovechan la riqueza del discurso como práctica social y el currículo como experiencia vivida. En tal sentido, otro aspecto relevante de este análisis es que 


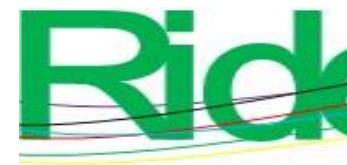

Revista Iberoamericana para la Investigación y el Desarrollo Educativo

ISSN 2007 - 7467

cuando se pesquisa la subjetividad a través huellas lingüísticas —desde una perspectiva sociocultural y fenomenológica - se acerca el análisis del discurso docente a la comprensión de la construcción de identidad y cultura curricular.

\section{Referencias}

Aoki, T. (2005). Teaching as Indwelling Between Two Curriculum Worlds. En Aoki, T., Pinar, W. and Irwin, R. Curriculum in a new key: the collected works of Ted T. Aoki. (pp. 159165). Nueva Jersey, Estados Unidos: Lawrence Erlbaum Associates/ Publishers.

Ávila, N. y Medina, L. (2012). El análisis dialógico del discurso (ADD) y la teoría de la enunciación: descubriendo la tensión dialógica en los discursos de profesores secundarios chilenos. Estudios de Psicología, 33(2), 231-247. Doi: 10.1174/021093912800676466.

Bajtín, M. (1981). The dialogic imagination. Texas, Estados Unidos: Prensa de la Universidad de Texas.

Bellei, C., \& Morawitz, L. (2016) Contenido fuerte, herramientas débiles. Las competencias del siglo XXI en la reforma educativa chilena. En F. Reimers \& C. Chung, Enseñanza y aprendizaje en el siglo XXI. Metas, políticas educativas y currículo en seis países (pp.121162). Ciudad de México: FCE.

Boschman, F., McKenney, S. and Voogt, J. (2014). Understanding decision making in teachers' curriculum design approaches. Educational Technology Research and Development, 62(4), 393-416.

Caro, M. y Aguilar, M. (2018). Desarrollo del currículum en las aulas: perspectivas del profesorado. En Arriata, A. y Osandón, L. (eds.), Políticas para el desarrollo del currículum. Reflexiones y propuestas (p. 337-381). Santiago, Chile: Ministerio de Educación.

Cox, C. (2018). Currículum: categorías de análisis, tendencias, gobernanza. En Arratia, A. y Osandón, L. (eds.), Políticas para el desarrollo del currículum. Reflexiones y propuestas (pp. 119-154). Santiago, Chile: Ministerio de Educación/ Unesco-Santiago.

Craig, C. J. (2012). Butterfly under a pin": An emergent teacher image amid mandated curriculum reform. The Journal of Educational Research, 105(2), 90-101. 


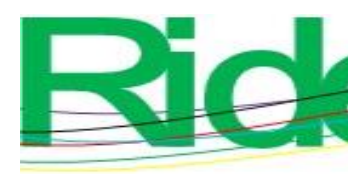

Revista Iberoamericana para la Investigación y el Desarrollo Educativo

ISSN $2007-7467$

Díaz Barriga, F. (2010). Integración de las TIC en el currículo y la enseñanza para promover la calidad educativa y la innovación. Pensamiento Iberoamericano, (7), 129-149.

Doyle, W. and Rosemartin, D. (2012). The ecology of curriculum enactment: Frame and task narrative. Interpersonal Relationships in Education, 137-147.

Espinoza, O., Riquelme, S. y Salas, A. (2017) Contextualización curricular (documento de trabajo de curso). Santiago, Chile: Pontificia Universidad Católica de Chile.

Greany, T. and Waterhouse, J. (2016). Rebels against the system: Leadership agency and curriculum innovation in the context of school autonomy and accountability in England. The International Journal of Educational Management, 30(7), 1188-1206. Retrieved from http://ezproxy.puc.cl/docview/1826443034?accountid=16788

Guzmán, M. (2011). Interacciones emergentes: una aproximación teórica para comprender el significado innovador de la producción curricular intra-aula. Pensamiento Educativo. Revista de Investigación Educacional Latinoamericana, 29(2), 319-340. Recuperado de http://pensamientoeducativo.uc.cl/files/journals/2/articles/208/public/208-481-1-PB.pdf

Guzmán, M., Meza, I., Pascual, E. y Pinto, R. (2007). La contextualización como estrategia de construcción curricular. Boletín de Investigación Educacional de la Facultad de Educación, PUCCH, 22(1), 209-230.

Handler, B. (2010). Teacher as curriculum leader: a consideration of the appropriateness of that role assignment to classroom based practioners. International Journal of Teacher Leadership, 3, 203-243.

Kerbrat-Orecchioni, C. (2010). S'adresser à autrui: les formes nominales d'adresse en français. París, Francia: Université de Savoie.

Larraín, A. y Medina, L. (2007). Análisis de la enunciación: distinciones operativas para un análisis dialógico del discurso. Estudios de Psicología, 28(3). Recuperado de http://www.dialogicidad.cl/papers/larrain.pdf

Magendzo, A. (2018). La educación del ciudadano: pensar un currículum para la vida democrática. En Arratia, A. y Osandón, L. (eds.), Políticas para el desarrollo del currículum. Reflexiones y propuestas (pp. 247-270). Santiago, Chile: Ministerio de Educación/ Unesco-Santiago.

Meneses, J. y Rodríguez, D. (2011). El cuestionario y la entrevista. Cataluña, España: UOC. 
Meza, I, Pascual, E. y Pinto, R. (2006). La contextualización como estrategia de construcción curricular. Boletín de Investigación Educacional de la Facultad de Educación, PUCCH, $22(1)$.

Pang, Y. (2012). Currículum development process. From Teaching to Knowledge, (3) 25-61.

Pinar, W. (2014). La teoría del curriculum. Ciudad de México, México: Narcea Ediciones.

Remillard, J. and Heck, D. (2014). Conceptualizing the curriculum enactment process in mathematics education. The International Journal on Mathematics Education, 46(5), 705 718.

Schiro, S. (2013). Curriculum Theory: Conflicting Visions and Enduring Concerns. California, Estados Unidos de América: SAGE Publications.

Schultz, B. D. (ed.). (2011). Listening to and learning from students: Possibilities for teaching, learning, and curriculum. IAP.

Shawer, S. (2010). Classroom-level curriculum development: EFL teachers as curriculumdevelopers, curriculum-makers and curriculum-transmitters. Teaching and Teacher Education, 26(2), 173-184. Doi: 10.1016/j.tate.2009.03.015

Voloshinov, V. (2009). El marxismo y la filosofía del lenguaje. Madrid, España: Alianza. 


\section{Anexo 1: Análisis dialógico del discurso por segmentos}

\section{Segmento 1 -inicio}

\section{Siglas:}

E: Entrevistador S: Sujeto entrevistado SE: Sujeto del enunciado E: Enunciador

\begin{tabular}{|l|l|}
\hline $\mathrm{N}^{\circ}$ línea & Transcripción \\
\hline 1 & I: Ya platicaste de la visión de la educación \\
2 & que tienes/ ahora ¿cómo es tu relación con el \\
3 & currículum?
\end{tabular}

$4 \quad$ S: A ver/ en cuanto como al CURRÍCULO

$5 \quad$ FORMAL a las BASES CURRICULARES

6 en sí / yo creo que antes era mucho más

7 apegada como cuándo uno está estudiando/

8 porque es como nuestra ley y la que rige

9 como los contenidos de las habilidades que

10 uno va a trabajar/ pero a medida que uno ya

11 se va desempeñando/ va conociendo a los

12 chiquillos /y uno también se va conociendo

13 /// Como como DOCENTE he logrado como

14 desapegarme y tomar como decisiones más

15 autónomas que también favorezcan el

16 cumplimiento de ese CONTENIDO Y

17 HABILIDADES que se solicita// La verdad

18 que trabajar con el currículo no me

19 complica// EL CURRÍCULUM también

20 opera de distintas formas/ está EL

21 CURRÍCULUM FORMAL/ está el currículo

22 oculto/ está EL CURRÍCULO INFORMAL

23 //Entonces la verdad es saber cómo en qué

24 momento o hacer la reflexión posterior/

25 porque como que cuando uno está haciendo

26 clases es muy difícil estar pensando cómo

27 está operando en ese momento y/ darse

28 cuenta quizás estoy trabajando mucho con

29 el CURRÍCULO INFORMAL y/ darse

30 cuenta y hacer como los ajustes necesarios porque también uno/ también tiene que volver a lo formal de cierta forma.

Mecanismos de inscripción

Modalización condicional: A ver

primera persona singular: yo (1)

primera persona indeterminado: Uno (6)

posesivo de la primera persona del plural: nuestro

Verbo pronominalizado: era/ estoy

Pronombre reflexivo primera persona:

$\mathrm{Me}$

Pronombre reflexivo tercera persona:

se

Verbo en voz pasiva: no me complica

Persona Singular del presente indicativo de haber: he logrado

Categorías pronominalizadoes:

Bases Curriculares/ los chiquillos/ el currículum/ el currículum formal/ el currículum informal/ el currículum oculto, contenido habilidades

Deítico de tiempo:

Antes, posterior

Modalidades:

Alética:

Ajustes necesarios

Epistémica: Yo creo que antes era más apegada

Deóntica: uno también tiene que volver a lo formal

Es nuestra ley, la que rige, favorezcan el cumplimiento

Favorezcan el cumplimiento de ese contenido y habilidades que se solicita

Epistemológica:

Entonces la verdad es saber cómo en qué momento o hacer la reflexión posterior

\section{Subjetivemas:}

Adjetivo afectivo: mucho más apegada / decisiones más autónomas

Adverbio: a medida, cuando estaba, cuando uno está haciendo clases es muy difícil/ trabajando mucho, quizás

Sujeto del enunciado: Flexibilidad de la profesora en relación con el uso del currículo formal.

E1: Me apego al currículo formal porque es nuestra ley.

E2: Me desapego al currículo formal porque me voy conociendo.

E2': Me desapego del currículo formal porque también existe en currículo formal e informal.

\section{Segmento 2- desarrollo}

Siglas:

E: Entrevistador S: Sujeto entrevistado SE: Sujeto del enunciado E: Enunciador

Nínea $\mid$ Transcripción

Mecanismos de inscripción 


\begin{tabular}{|l|l|l|}
\hline 1 & E: Y en la institución ¿qué tanta flexibilidad & Pronombre primera persona del plural: nos \\
2 & hay para esto? Si yo veo que las Bases & $\begin{array}{l}\text { Verbo pronominalizado primera persona del } \\
\text { plural: vamos, tenemos, teníamos }\end{array}$ \\
3 & curriculares exigen algo y el objetivo me & Pronombre relativo terecra persona: lo \\
4 & queda grande chico ¿hay posibilidades de que & Categoría nominal: \\
5 & yo me salga de ahí? & Institución/ Bases curriculares, niños, niñas, objetivo, \\
6 & S: Por un tema como legal todo tiene que & la autonomía \\
7 & tributar al objetivo de aprendizaje/ porque & Modalidad \\
8 & supuestamente es lo mínimo que los NIÑNOS & Deóntica: \\
9 & y NINAS tienen que aprender// Si nos puede & Todo tiene que tributar al objetivo \\
10 & pasar/ que un OBJETIVO lo teníamos & Tienen que aprender \\
11 & calendarizado para que se desarrollara en diez & Autonomía \\
12 & horas pedagógicas/ y con la trayectoria y las & Alética: \\
13 & evaluaciones formativas nos damos cuenta & Vamos a necesitar \\
14 & de que la verdad vamos a necesitar más & Si nos puede pasar \\
horas o menos horas/ sí tenemos LA & Que sería como \\
AUTONOMÍA de hacer esos como ajustes/ & Epistemológico: \\
& que sería también solo como de forma // & Nos damos cuenta \\
& \multicolumn{2}{|l|}{ Subjetivema: } \\
\hline
\end{tabular}

Segmento 3-desarrollo

Siglas:

E: Entrevistador S: Sujeto entrevistado

\begin{tabular}{|l|l|l|}
\hline$N^{\circ}$ línea & Transcripción & Mecanismos de inscripción \\
\hline 1 & E: Y ese enfoque/ sobre el uso para la vida & Pronombre indeterminado primera persona: Uno \\
2 & icrees que se refleja en las bases & 6) \\
3 & curriculares? & Verbo pronominalizado de a primera persona: \\
4 & S:Como que en teoría las Bases también & Intento \\
5 & emm como que explicitan eso/ como que en & Verbo pronominalizado reflexivo tercera persona: \\
6 & la introducción de las BASES & se \\
7 & CURRICULARES también de cierta forma & Verbos en voz pasiva: se te exige \\
8 & están basadas en que tenga un sentido y & Categorías nominales: \\
9 & que tenga un aprendizaje significativo/ & Las Bases curriculares, el colegio, el contenido, el \\
10 & pero también hay muchos objetivos/ que es & contenido de historia \\
11 & muy complejo hacer eso/ como ese vuelco/ & Deítico demostrativo: \\
12 & que supuestamente las bases piden. Pero & Eso (2), esa. \\
13 & también el contenido a veces que uno tiene & Subjetivemas \\
14 & que pasar/ uno no siempre logra darle esa/ & Adverbio: Supuestamente las bases piden \\
15 & Adjetivos de cantidad: Muchos objetivos/ Historia es \\
16 & como ponerle ese sello a la unidad porque & muy grande/ muy amplio/ muy complejo hacer eso \\
17 & también uno tiene que cumplir con lo que & Adverbio de tiempo: \\
18 & se te exige /I/ como que uno tiene que & No siempre se logra \\
19 & cumplir sí o sí con las Bases y a veces es & siempre intento darles sentido a las clases. \\
20 & mucho CONTENIDO// Por ejemplo 6 & Adjetivo Afectivo: uno se ve como cuartado/ un poco \\
21 & básico EL CONTENIDO DE HISTORIA es & Adverbio relativo: \\
22 & muy grande es muy amplio// Y la verdad & Como que (4), Como ese, de cierta forma \\
23 & como que uno tiene que ir avanzando con & Modalidad \\
\hline
\end{tabular}




\begin{tabular}{|l|l|l|}
\hline 24 & las evaluaciones con lo que te pide el colegio & Alética: \\
25 & entonces a veces uno se ve como coartado/ & Que tenga un sentido y que tenga un aprendizaje \\
26 & un poco/ perol pero siempre intento darle & significativo \\
27 & sentido a las clases. & Deóntico: \\
28 & & El contenido que uno tiene que pasar \\
29 & uno tiene que cumplir \\
30 & uno tiene que cumplir si so si con las bases \\
31 & Espistémica: \\
32 & La verdad como que uno tiene que ir avanzando \\
33 & Volitiva: \\
34 & Siempre intento darle sentido \\
35 & Conjunciones \\
36 & Pero también hay muchos objetivos \\
37 & & Pero también el contenido a veces que uno tiene que \\
38 & pasar \\
39 & Pero siempre intento darle sentido a las clases \\
\end{tabular}

\section{Segmento 4- cierre}

\begin{tabular}{|l|l|l|}
\hline $\mathrm{N}^{\circ}$ línea & Transcripción & Mecanismos de inscripción \\
\hline 1 & I: Perfecto/ ok. Pues no sé algo más que quieres & Verbo pronominalizado primera persona: estoy \\
2 & agregar que quieres comentar me quedo con & Pronombre Posesivo primera persona: mi \\
3 & estas tensiones y que en medio de la tensión la & Pronombre indeterminado primera persona: uno \\
4 & institución de la política hay pequeños espacios & Categorías nominales: \\
5 & S: Por lo menos en la institución que estoy EL & El aula, la actividad, el ministerio, la planificación \\
6 & AULA es mi espacio de toma de DECISIÓN & anual \\
7 & I: ¿inclusive más que en la planificación? & Subjetivema: \\
8 & S: En LA PLANIFICACIÓN ANUAL no hay & Adverbio: no hay mucho que tomar decisiones \\
9 & mucho que tomar decisiones/ porque es como & Adverbio de modo: es netamente de uno \\
10 & ordenar los objetivos para formar unidades y & Adverbio de tiempo: entonces, muchas veces \\
11 & muchas veces están dados también desde EL & por lo menos (salvedad de lo que se acaba de decir) \\
12 & MINISTERIO/ entonces puede ser como un & Modalidad: \\
13 & copy paste. Pero si en lo que es de la clase a & Alética: \\
14 & clase como en LA ACTIVIDAD que voy a & puede ser como un copy paste \\
15 & realizar para lograr ese OBJETIVO eso es & volitiva: \\
16 & netamente de uno. & voy a realizar para lograr ese objetivo \\
\end{tabular}

